\title{
Estilo pictórico em animações em saúde: uma análise de casos clínicos da UNA-SUS/UFMA
}

\author{
Picture style in animations in health: an analysis of clinical cases \\ of UNA-SUS / UFMA
}

\author{
Camila Santos de Castro e Lima, Carla Galvão Spinillo
}

animação, estilo pictórico, caso clínico, saúde

\begin{abstract}
Animações de casos clínicos são utilizadas no ensino a distância em saúde para representar situações atuais ou fictícias, auxiliando profissionais na tomada de decisões médicas. No Brasil, a Universidade Aberta do SUS da UFMA (UNA-SUS/UFMA) utiliza amplamente animações de casos clínicos em seus cursos a distância. No entanto, a qualidade gráfica e de comunicação de suas animações carece de estudos. Assim, este artigo apresenta uma análise de estilo pictórico de uma amostra de seis animações de casos clínicos produzidas pela UNA-SUS/UFMA. Os resultados mostraram que as animações possuem poucas variações de técnicas e detalhes nas ilustrações, o arranjo dos elementos do contexto pictórico tem, em certa medida, aleatoriedade, a maioria das animações carece de simulação de movimento e detalhes que produzem maior realismo à representação de conteúdos. O uso de recursos enfáticos que pudessem facilitar a aprendizagem em aspectos de atenção, percepção, processamento de informações e memória, não foram identificados nas animações. Essas fragilidades podem comprometer a clareza, estrutura, harmonia e proporção pictórica das animações analisadas. Por fim, infere-se que as características do estilo pictórico em animações de casos clínicos merecem maior atenção em relação à adequação e aceitação da representação dos conteúdos direcionados aos usuários/profissionais de saúde.
\end{abstract}

animation, pictorial style, clinical case, health

Animations of clinical cases have been used in distance learning in health to represent actual or fictitious situations, assisting professionals in making medical decisions. In Brazil, the Open University of SUS of UFMA (UNA-SUS/UFMA) widely uses animations of clinical cases in their distance learning courses. However, the graphic and communication quality of their animations lack studies. Thus, this article presents a pictorial style analysis of a sample of six animations of clinical cases produced by UNASUS/UFMA. The results showed that the animations have few variations of techniques and detail in the illustrations, the arrangement of the elements of the pictorial context is to a certain extent randomness, most animations lack simulation of movement and details that produce greater realism to the representation of contents. The use of emphatic resources that could facilitate learning, in aspects of attention, perception, information processing and memory, were not identified in the animations. These drawbacks may compromise the clarity, structure, harmony, and pictorial proportion of the animations analyzed. Finally, it is inferred that the characteristics of pictorial style in animations of clinical cases deserve further attention in relation to the adequacy and acceptance of the representation of contents addressed to users /health professionals.

Anais do $10^{\circ} \mathrm{CIDI}$ e $10^{\circ} \mathrm{CONGIC}$

Kelli C.A.S. Smythe, Rafael de Castro Andrade (orgs.)

Sociedade Brasileira de Design da Informação - SBDI

Curitiba | Brasil | 2021
Proceedings of the $10^{\text {th }} \mathrm{CIDI}$ and $10^{\text {th }}$ CONGIC

Kelli C.A.S. Smythe, Rafael de Castro Andrade (orgs.)

Sociedade Brasileira de Design da Informação - SBDI Curitiba | Brazil | 2021 


\section{Introdução}

No contexto educacional, especialmente na Educação a Distância ( $E a D)$ torna-se importante o desenvolvimento de novas abordagens e recursos educacionais para promover eficácia no processo de ensino-aprendizagem. Para isto, têm sido desenvolvidas diversas formas de apresentação de conteúdos, como e-books, jogos, infográficos, animações. As animações em específico possuem vantagens em relação à apresentação de situações abstratas, processos e procedimentos, por exigirem um menor esforço cognitivo e de maneira mais confortável para o usuário (e.g., Ainsworth, 2008; Lowe \& Scnhotz, 2008; Lima et al., 2019; Andrade, 2014).

$\mathrm{Na}$ área da saúde, animações são empregadas para representar casos clínicos mostrando situações médicas reais ou fictícias, visando treinar profissionais de saúde na tomada de decisão sobre pacientes, contextos de saúde, procedimentos, diagnósticos e tratamentos médicos. Isto atesta o papel de destaque na animação com esta finalidade. Casos clínicos simulados tornam-se recursos relevantes na capacitação de profissionais de saúde para sua atuação em situação real, particularmente na EaD em saúde.

No ensino a distância na educação continuada de profissionais de saúde no Brasil, tem-se a Universidade aberta do Sistema Único de Saúde (UNA-SUS), formada por uma rede de instituições federais de ensino superior. É um projeto do Ministério da Saúde, desenvolvido pela Secretaria de Gestão do Trabalho e da Educação na Saúde - SGTES. Dentre os membros da rede UNA-SUS, destaca-se a Universidade Federal do Maranhão (UNASUS/UFMA) por ser uma das primeiras a integrar a rede e por ter produção significativa de recursos educacionais e cursos. Ela atua desde 2009 oferecendo cursos a distância de capacitação, especialização e extensão para mais de 550 mil profissionais da área da saúde que atuam no SUS em todo o país (UNA-SUS/UFMA, 2021). A UNA-SUS/UFMA emprega em seus diversos cursos, animações de casos clínicos em saúde desenvolvidas por equipes multidisciplinares. Apesar da importância das animações de casos clínicos como recursos educacionais da UNA-SUS/UFMA, não se tem estudos que investiguem sua qualidade gráfica e comunicacional.

Portanto, torna-se pertinente estudos em design da informação sobre uso de animação em casos clínicos, enfocando aspectos de seu design, como por exemplo o estilo de ilustração empregado. Este tem sido estudado em representações estáticas (e.g. Lopes \& Spinillo, 2017; Oliveira \& Coutinho, 2017) indicando sua relevância para o desenvolvimento e compreensão de ilustrações, ou seja, de representação pictórica de conteúdos. Isto é particularmente importante para a área de saúde visto que se as ilustrações em casos clínicos apresentarem deficiências em seu design, podem vir a comprometer a visualização do conteúdo representado, e consequentemente a sua compreensão. Assim sendo, a tomada de decisão do profissional de saúde em capacitação estaria prejudicada.

Considerando tais aspectos, este artigo apresenta uma análise de estilo pictórico em animações de casos clínicos médicos. Para isso foram selecionadas animações produzidas pela UNA-SUS/UFMA. A seguir são apresentados alguns conceitos e abordagens de design da informação que fundamentaram a análise das animações a ser explicitada posteriormente. 


\section{Animações e casos clínicos em saúde}

As animações no contexto da educação podem ser utilizadas para representar situações abstratas, instruções, conteúdos, processos e procedimentos, de uma forma mais confortável e que exija menos esforço cognitivo (Lima et al., 2019). Em seu estudo sobre infografia na área de saúde, Andrade (2014) ressalta algumas das vantagens do uso das animações, sendo elas:

- Representar situações abstratas ou que não sejam facilmente vistas no dia-a-dia, tais como fórmulas matemáticas ou movimento de moléculas;

- Facilitar a visualização e compreensão de processos/procedimentos;

- Reduzir o esforço cognitivo empregado para o processamento da informação;

- Motivar o aprendizado;

- Os indivíduos percebem as animações como a forma mais confortável para receber instruções de como realizar uma tarefa.

No que concerne animações de casos clínicos, estas são utilizadas como forma de apresentar situações (que podem ser fictícias ou não), para que o profissional observe, analise e entenda a situação apresentada, de forma que possa adquirir conhecimento de experiência para tomada de decisão em uma situação real (Galdeano, Rossi \& Zago, 2003).

Segundo Galdeano, Rossi e Zago (2003), o caso clínico deve ser elaborado levando em consideração aspectos de contextualização, fundamentação teórica, questionamentos e soluções, à partir das seguintes etapas: (1) Definição das questões norteadoras; (2) Identificação do local e/ou pessoa em estudo; (3) Resumo dos problemas ou alterações identificados; (4) Fundamentação teórica; (5) Alternativas ou propostas; (6) Ações para implementação ou recomendações; (7) Discussão. Desta forma, a animação seria o recurso educacional que materializa estas etapas, podendo ser considerada como o artefato gráfico educacional resultante deste processo (Figura 1).

Figura 1: Roteiro de desenvolvimento de casos clínicos e animação como artefato gráfico educacional resultante com base em Galdeano, Rossi e Zago, 2003. Fonte: as autoras.

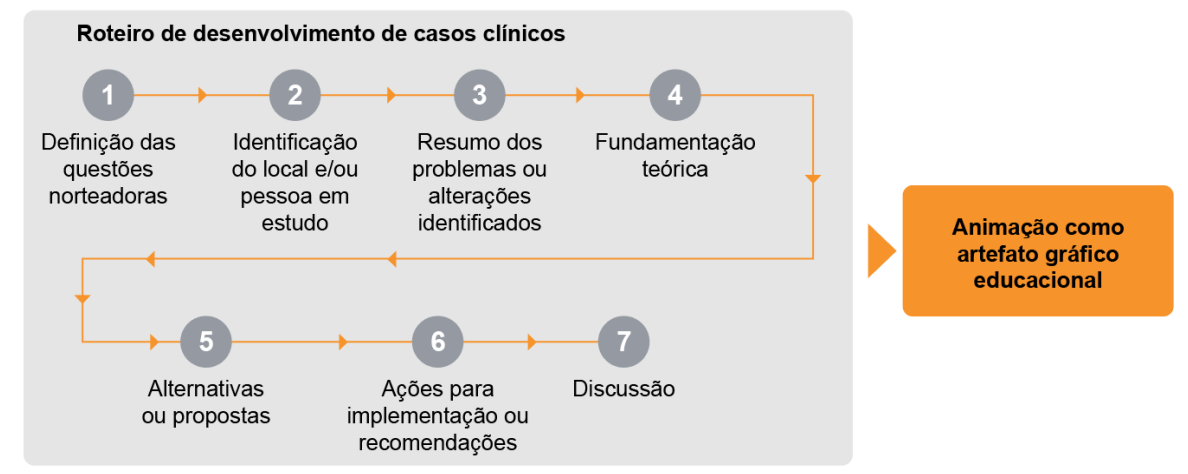

A elaboração de animações de casos clínicos, por sua vez, se dá em um processo de tomada de decisões não apenas tecnológicas, mas também estilísticas em seu design. 
Segundo Ashwin (1979), ao desenvolver uma ilustração, o profissional continuamente toma decisões - seja de forma consciente ou quase de maneira reflexiva - que culminam na determinação do caráter estilístico da ilustração. Este tópico é apresentado a seguir.

\section{Estilo pictórico e princípios de design da informação}

Levando em conta os aspectos estilísticos das ilustrações das animações de casos clínicos, aqui serão tratados conceito, e modelo descritivo de estilo de ilustração proposto por Ashwin (1979). Além disto são colocados os princípios de design propostos por Pettersson (2007), os quais tornam-se pertinentes para elaboração de inferências sobre possíveis efeitos do estilo pictórico na comunicação de conteúdos nas animações de casos clínicos analisadas.

\section{Modelo descritivo de estilo em ilustrações}

Segundo Gombrich (1995), o estilo pode ser compreendido como características que diferenciam as representações pictóricas, que objetivam agrupar, organizar e identificar as imagens e ilustrações a partir de distinções de técnicas utilizadas, podendo estar relacionado a diversas ideias, temperamento e personalidade, preferências de pessoas ou artistas, até mesmo movimentos, escolas de arte ou períodos históricos. Para Dondis (1997) as variantes técnicas podem ser utilizadas para identificar a individualidade estilística, distinguindo estilos e períodos das representações. Twyman (1985) por sua vez, afirma que o estilo tem relação com os meios de produção, formas, recursos, disposição, contendo diversos tipos de representação como em horizontais, verticais, relação espacial, contraste de tons e outras categorias.

Por fim, Ashwin (1979) considera estilo 'ingredientes gráficos' de imagens/ilustrações e para isto estabelece sete variáveis. Estas compõem seu modelo descritivo adotado no presente artigo. Para cada variável são atribuídos dois polos que definem a 'força' da variável, sendo estas:

1. A Consistência refere-se às técnicas de representação e às ferramentas utilizadas para elaborar uma determinada ilustração. Seus polos são: Homogêneo, quando não tem técnicas variadas de produção (por exemplo, um desenho a traço); Heterogêneo, quando possui variedade de técnicas de produção e ainda mistura de imagens e textos (desenhos, fotografia, colagem, etc.);

2. A Gama possui relação com o nível de detalhes dos elementos que compõem uma ilustração. Os seus polos são: Contraído, com poucos detalhes; Expandido, com muito detalhamento, utilizando diversas possibilidades sintáticas;

3. O Enquadramento relaciona-se à disposição da imagem pictórica e seu suporte. Seus polos são: Conjuntivo, onde o contexto está disposto em volta da figura principal (cenários por exemplo); Disjuntivo, quando o usuário cria o contexto;

4. Já o Posicionamento refere-se à composição da ilustração, podendo ser Simétrico, transmitindo ordem, ou Casual, sugerindo uma certa aleatoriedade, ou ainda com variantes de proporção e tamanho dos elementos; 
5. A Proximidade é uma variável que tem relação com a proximidade ou distância entre a representação e o usuário, ou seja, com a escala da figura. Tem como polos Perto (por exemplo o desenho de um busto) e Distante;

6. A variável Cinética, caracteriza-se pela sugestão de movimento implícito na imagem e tem como polos Estático e Dinâmico. O movimento em uma imagem estática pode ser sugerido a partir de linhas de velocidade ou uma sequência de um mesmo personagem em posições diferentes, por exemplo;

7. Por fim, o Naturalismo, tem relação com a veracidade da imagem, ou seja, com o grau de realismo da ilustração, a relação física do objeto representado com o mundo real. Possui polos Naturalista e Não-naturalista

O quadro 1 sumariza estas variáveis e seus polos. Cada variável é apresentada em uma cor como forma de diferenciá-la graficamente. Uma breve descrição de cada variável é também colocada no quadro.

Quadro 1: Polos e variáveis de estilos pictóricos de acordo com Ashwin (1979). Fonte: as autoras

\begin{tabular}{|c|c|c|}
\hline Polo & Variável & Polo \\
\hline Homogênea & Consistência & Heterogênea \\
\hline \multicolumn{3}{|c|}{$\begin{array}{l}\text { Refere-se às técnicas de representação e às ferramentas } \\
\text { utilizadas para elaborar uma determinada ilustração. }\end{array}$} \\
\hline Contraído & Gama & Expandido \\
\hline \multicolumn{3}{|c|}{$\begin{array}{l}\text { Possui relação com o nível de detalhes dos elementos que } \\
\text { compõem uma ilustração }\end{array}$} \\
\hline Conjuntivo & Enquadramento & Disjuntivo \\
\hline \multicolumn{3}{|c|}{$\begin{array}{l}\text { Relaciona-se à disposição da imagem pictórica e seu } \\
\text { suporte. }\end{array}$} \\
\hline Simétrico & Posicionamento & Casual \\
\hline \multicolumn{3}{|c|}{ Refere-se a composição da ilustração. } \\
\hline Perto & Proximidade & Distante \\
\hline \multicolumn{3}{|c|}{$\begin{array}{l}\text { Tem relação com a proximidade ou distância entre a } \\
\text { representação e o usuário, ou seja com a escala da figura. }\end{array}$} \\
\hline Estático & Cinética & Dinâmico \\
\hline \multicolumn{3}{|c|}{$\begin{array}{l}\text { Caracteriza-se pela sugestão de movimento implícito na } \\
\text { imagem. }\end{array}$} \\
\hline Naturalista & Naturalismo & Não-naturalista \\
\hline \multicolumn{3}{|c|}{$\begin{array}{l}\text { Tem relação com a veracidade da imagem, ou seja com o } \\
\text { grau de realismo da ilustração, a relação física do objeto } \\
\text { representado com o mundo real }\end{array}$} \\
\hline
\end{tabular}


Vale salientar que as variáveis propostas por Ashwin (1979) foram consideradas para ilustrações impressas, portanto estáticas. Porém, como o estilo pictórico independe da ilusão de movimento imposta em animações, o uso do modelo de Ashwin torna-se pertinente, mesmo considerando a variável 'Cinética', conforme será visto na análise das animações de casos clínicos.

\section{Princípios de design da informação (Pettersson, 2007)}

Pettersson (2007), propõe princípios de design da informação para projetar materiais eficazes em comunicação de mensagens. Estes princípios estão categorizados em: funcionais, estéticos, cognitivos e de gestão (Figura 2).

Figura 2: Princípios de design baseados em Pettersson (2007). Fonte: as autoras

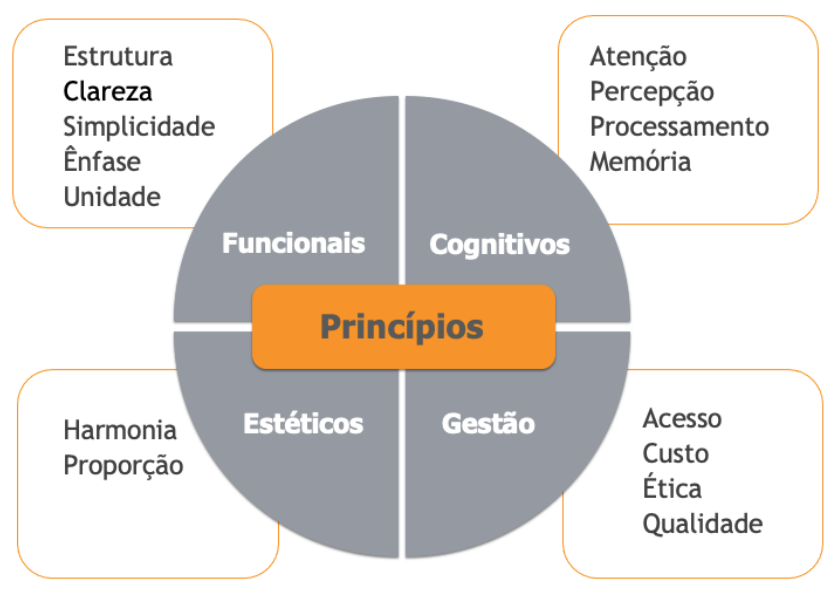

Para fins deste artigo, o foco será nos princípios funcionais, estéticos e cognitivos, por serem aqueles que possuem relação direta com a representação de uma informação. Sendo assim, os princípios funcionais são:

- Definição do problema: identificar, analisar e organizar a forma como o produtor da mensagem, o receptor, o material e o contexto em que a comunicação ocorre;

- Estrutura: deve-se desenvolver uma estrutura organizada e limitada que possua hierarquização do conteúdo, de uma forma que facilite a interação e a compreensão do usuário em relação ao material;

- Clareza: referente à legibilidade e apresentação não ambígua, evitando elementos ou detalhes que não sejam relevantes para as representações pictóricas;

- Simplicidade: deve-se manter uma consistência nos materiais priorizando as informações visuais relevantes, a fim de promover uma maior efetividade em relação a percepção, processamento e memória;

- Ênfase: consiste em utilizar elementos para enfatizar informações importantes, direcionando a atenção do usuário. Pode-se utilizar áreas de cores, sombras, contraste, destacar com formas o objeto, fazer uso de movimento, etc. 
- Unidade: refere-se à coerência e consistência do artefato como um todo. Materiais que são inconsistentes podem confundir os usuários.

Já os princípios estéticos são:

- Harmonia: refere-se aos elementos estarem visualmente agradáveis quando colocados juntos. A falta deste princípio pode gerar distração;

- Proporção: refere-se às proporções entre os elementos, sem excessos visuais, fazendo com que o usuário tenha um interesse pela apresentação gráfica do material.

Por fim, os princípios cognitivos são:

- Facilitar a atenção: utilizar recursos que direcionam a atenção do usuário, através de imagens interessantes, utilizando elementos como setas, ícones, cores, linhas, etc.

- Facilitar a percepção: utilizar recursos que auxiliem na detecção e identificação de informações, como por exemplo utilizar o tipo de representação adequado para uma situação (fotografia, imagens 2D, 3D), com conteúdos interessantes e que tenham um bom contraste entre a figura e o fundo;

- Facilitar o processamento mental: com recursos que facilitem o fluxo da informação para o entendimento da mensagem, sendo consistente nos materiais, utilizando um número limitado de elementos gráficos e focando no que é relevante em relação ao conteúdo;

- Memória: utilizar recursos que promovam a retenção e o armazenamento de informações, com o uso limitado de elementos ao mesmo tempo, conteúdos significativos e ainda conexão entre os elementos apresentados no material.

As variáveis de estilo de ilustração (Ashwin,1979) e princípios de design da informação (Pettersson, 2007) foram norteadores da análise das animações de casos clínicos em saúde da UNA-SUS/UFMA, a qual é apresentada a seguir.

\section{Análise das animações de casos clínicos da UNA-SUS/UFMA}

A análise das animações de casos clínicos em saúde da UNA-SUS/UFMA visou identificar possíveis comunalidades em relação ao(s) estilo(s) pictórico(s) destes recursos e inferir sobre influências dos aspectos estilísticos identificados quanto à eficácia comunicativa junto a seus usuários/profissionais de saúde.

\section{Material e procedimento}

Para a seleção das animações realizou-se uma busca empregando as palavras-chaves "caso" e "clínico", no canal próprio da UNA-SUS/UFMA no YouTube (https://www.youtube.com/channel/UCPTXC-pzmpYRS3k1dLu1v4g) que possui 19,5 mil inscritos. Como resultado desta etapa foram identificadas um total de 86 animações. O critério 
de inclusão da amostra foi 'aquelas produzidas entre os anos de 2016 e 2021', resultando em 24 animações. Por fim, excluiu-se as animações que possuíam repetição de personagens, com as mesmas características gráficas e/ou estavam divididas em sequências ou partes, como por exemplo: parte 1, parte 2 e parte 3 do mesmo caso clínico. Isto resultou em seis animações para este estudo. O quadro 2 mostra os títulos, anos e links das animações da UNASUS/UFMA disponíveis até esta data no YouTube.

Quadro 2: Animações de casos clínicos analisadas. Fonte: as autoras

\begin{tabular}{lcl}
\hline Título & Ano & Link \\
\hline Caso de Sr. João & 2019 & $\begin{array}{l}\text { https://www.youtube.com/watch?v=EG2- } \\
\text { mIA5hj\&ab_channel=UNA-SUS\%2FUFMA }\end{array}$ \\
\hline Avaliação entre pares: caso Rio de Janeiro & 2017 & $\begin{array}{l}\text { https://www.youtube.com/watch?v=IdMN3vTRtkI\&a } \\
\text { b_channel=UNA-SUS\%2FUFMA }\end{array}$ \\
\hline Caso Cláudio: vídeo l & 2017 & $\begin{array}{l}\text { https://www.youtube.com/watch?v=NI1KjHyel60\&a } \\
\text { b_channel=UNA-SUS\%2FUFMA }\end{array}$ \\
\hline $\begin{array}{l}\text { Cidade de Samambaia: curso de } \\
\text { leishmaniose visceral - Vigilância em Saúde }\end{array}$ & 2017 & $\begin{array}{l}\text { https://www.youtube.com/watch?v=kRLPvdrTyMI\& } \\
\text { ab_channel=UNA-SUS\%2FUFMA }\end{array}$ \\
\hline $\begin{array}{l}\text { DRC - Atendimento interdisciplinar ao } \\
\text { paciente - parte 1 }\end{array}$ & 2016 & $\begin{array}{l}\text { https://www.youtube.com/watch?v=4G8dqkfAoiM\& } \\
\text { ab_channel=UNA-SUS\%2FUFMA }\end{array}$ \\
\hline Caso Clínico Prevenção em DRC & 2016 & $\begin{array}{l}\text { https://www.youtube.com/watch?v=CLdmGIR3U2c } \\
\text { \&ab_channel=UNA-SUS\%2FUFMA }\end{array}$ \\
\hline
\end{tabular}

Realizou-se a análise das animações através da aplicação do protocolo descritivo das características do estilo gráfico das imagens desenvolvido por Oliveira e Coutinho (2017) com base em Ashwin (1979). Este consta de nove gradientes entre os polos, sendo quatro em direção a cada polo e o gradiente do meio referindo-se ao ponto neutro (zero) entre os polos. A escolha deste protocolo com adaptação do modelo de Ashwin se deu por considerar que um maior número de gradientes permite uma análise mais detalhada do estilo pictórico nas animações. Isto foi argumentado também pelas autoras que propuseram a adaptação (Oliveira \& Coutinho, 2017) quando analisando ilustrações de um artista gráfico em Pernambuco. O quadro 3 mostra o protocolo utilizado na análise das animações de casos clínicos.

Quadro 3: Protocolo de análise do estilo das animações. Fonte: Adaptado de Oliveira \& Coutinho, 2017.

\begin{tabular}{|c|c|c|c|c|c|c|c|c|c|c|c|}
\hline & & 4 & 3 & 2 & 1 & 0 & 1 & 2 & 3 & 4 & \\
\hline Consistência & Homogênea & & & & & & & & & & Heterogênea \\
\hline Gama & Contraido & & & & & & & & & & Expandido \\
\hline Enquadramento & Conjuntivo & & & & & & & & & & Disjuntivo \\
\hline Posicionamento & Simétrico & & & & & & & & & & Casual \\
\hline Proximidade & Perto & & & & & & & & & & Distante \\
\hline Cinética & Estático & & & & & & & & & & Dinâmico \\
\hline Naturalismo & Naturalista & & & & & & & & & & Não-naturalista \\
\hline
\end{tabular}


A análise foi conduzida pelas autoras deste trabalho, empregando-se o protocolo adaptado de Ashwin em cada animação de caso clínico, e em seguida foi realizada uma compilação dos resultados em uma tabela a fim de verificar as comunalidades no estilo pictórico. A análise se deu de forma qualitativa, pelo pequeno número de animações de casos clínicos. A síntese dos resultados é apresentada a seguir, sendo estes discutidos com base nos princípios de design da informação propostos por Pettersson (2007). Desta forma, realizou-se uma análise heurística com base nos princípios de design da informação. Entretanto, destaca-se a necessidade de realizar estudos com usuários.

\section{Síntese dos resultados e discussão}

De forma geral, pode se dizer que a análise das animações de casos clínicos indica que estas possuem comunalidades em três das sete variáveis gráficas de estilo pictórico: consistência homogênea $(n=4 / 6)$, cinética estática $(n=5 / 6)$ e sendo também não-naturalistas $(n=4 / 6)$. Vale salientar que, a cinética foi considerada 'estática' na animação quando os elementos em tela (e.g., paciente, médico) não apresentavam movimento, mas sim ocorria sua 'aparição' na tela, o que foi comum. Por sua vez, as animações foram consideradas não-naturalísticas ainda que todas representassem conteúdos factíveis/verdadeiros, ou seja, possíveis de acontecer na realidade médica. O naturalismo foi avaliado em relação à veracidade ou acurácia pictórica da animação, ou seja, o quanto as imagens apresentavam ou não grau de detalhe necessário para representação do referente. Os resultados ainda mostraram que metade das animações $(n=3 / 6)$ apresentava-se neutra quanto à proximidade com o usuário, o que pode ser relacionado ao plano de câmera médio. As demais características de estilo ocorreram de forma variada em incidência nos seus polos, porém indicando tender para alguns dos polos. Assim, pode se dizer que as animações tendem a ter gama contraída, enquadramento conjuntivo e posicionamento casual. A síntese dos resultados encontra-se no quadro 4 e comentários sobre cada variável são apresentados a seguir.

Quadro 4: Síntese das análises das animações. Fonte: as autoras.

\begin{tabular}{|c|c|c|c|c|c|c|c|c|c|c|c|}
\hline & & 4 & 3 & 2 & 1 & 0 & 1 & 2 & 3 & 4 & \\
\hline Consistência & Homogênea & 4 & & & & & 1 & 1 & & & Heterogênea \\
\hline Gama & Contraído & 2 & 2 & 1 & & & 1 & & & & Expandido \\
\hline Enquadramento & Conjuntivo & 1 & 1 & 2 & & & & & 1 & 1 & Disjuntivo \\
\hline Posicionamento & Simétrico & 1 & 1 & & & & 2 & 1 & & 1 & Casual \\
\hline Proximidade & Perto & & & 1 & & 3 & & & 1 & 1 & Distante \\
\hline Cinética & Estático & 5 & 1 & & & & & & & & Dinâmico \\
\hline Naturalismo & Naturalista & & & & & & & & 2 & 4 & Não-naturalista \\
\hline
\end{tabular}

Em relação ao valor da Consistência, percebeu-se uma predominância do polo Homogêneo $(n=4)$, ou seja, sem muitas variações em relação às técnicas de elaboração das 
ilustrações. Identificou-se o uso de ilustrações 2D, produzidas por computador, em vetor, sem muito detalhamento, sombra e contraste. Apesar da não variação de técnicas manter a simplicidade e unidade do material, com uma coerência e consistência visual, levando até mesmo a uma certa harmonia, deve-se destacar que dependendo da proposta pedagógica, utilizar outros tipos de representação.

A Gama com tendência a ser Contraída (somando $n=5$ ) por pouco detalhamento dos personagens, objetos e cenários, o que pode interferir na percepção e atenção dos usuários em relação ao conteúdo apresentado, principalmente por não apresentar um bom contraste entre a figura e o fundo, podendo atrapalhar a clareza, detecção e identificação dos elementos das ilustrações, bem como causar aos usuários algum tipo de estranheza ou distração. No exemplo a seguir, percebe-se o uso de ilustrações em estilo 2D, sem variação de técnicas, sem detalhamento, com pouco contraste entre os objetos, personagens e cenários, sem utilizar ênfase para destacar pontos relevantes para a narrativa do caso clínico.

Figura 3: Capturas de tela do caso: Caso Sr. João. Fonte: UNA-SUS/UFMA, 2019.

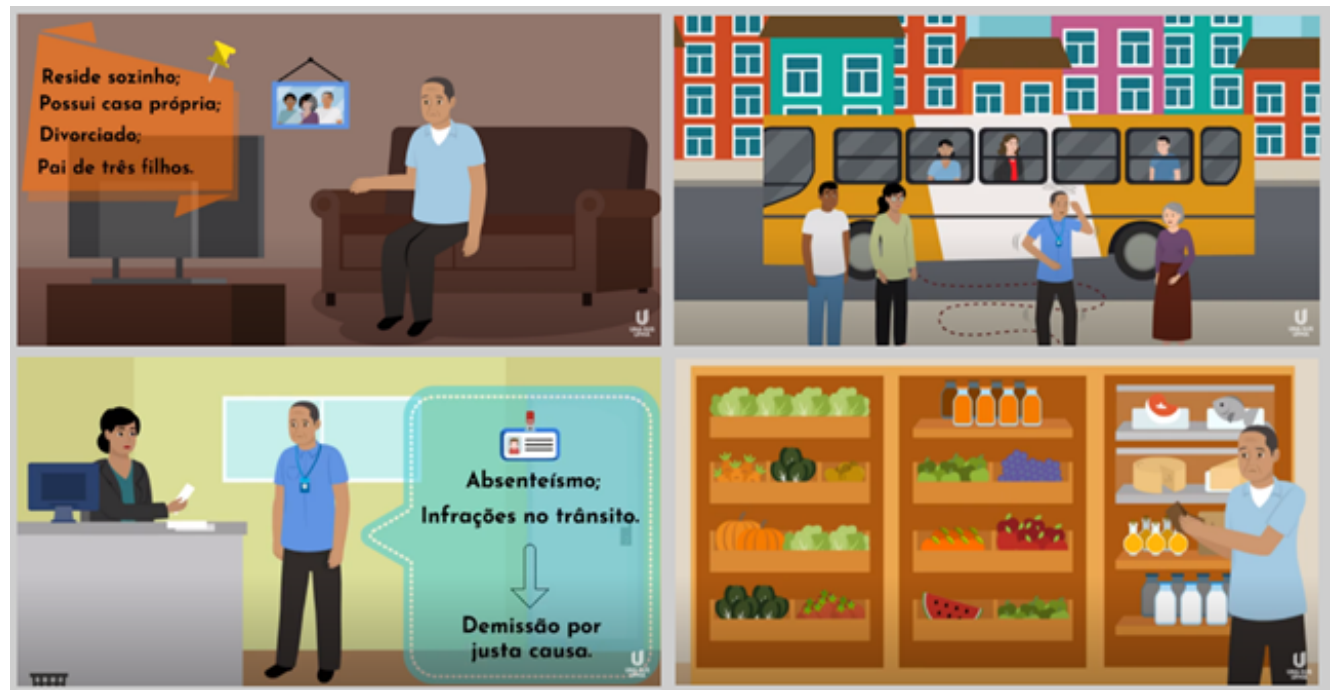

O Enquadramento foi identificado como Conjuntivo $(n=4)$, ou seja, com o contexto disposto em volta da figura principal, ainda que em algumas animações os cenários não fossem tão explorados (sem muito detalhamento ou utilizando uma única cor, por exemplo). Neste caso, pela mesma questão anterior, percebe-se que os personagens e cenários acabam não tendo uma diferenciação, o que atrapalha a clareza, bem como a estrutura, atenção e percepção. Além disso, pode atrapalhar a harmonia e proporção dos elementos nas ilustrações, tendo em vista que os elementos das imagens confundem-se e por vezes acabam parecendo um único objeto, conforme pode ser visto no exemplo a seguir. 
Figura 4: Capturas de tela do caso: Cidade de Samambaia: curso de leishmaniose visceral Vigilância em Saúde. Fonte: UNA-SUS/UFMA, 2017.

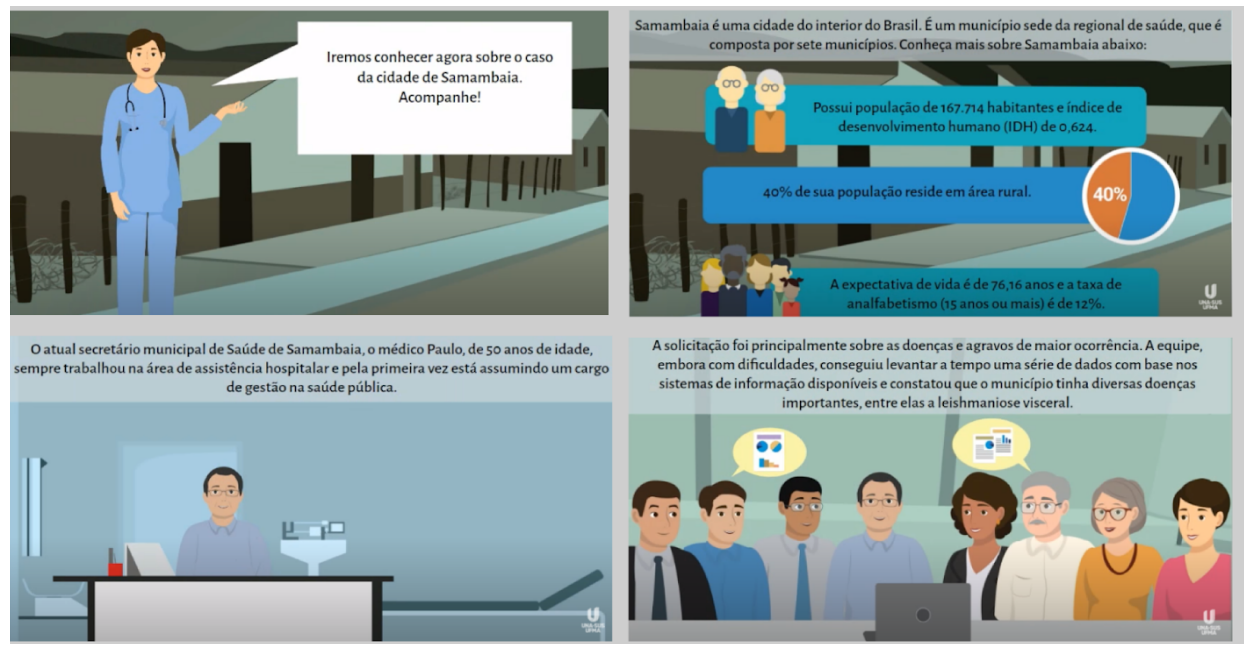

O Posicionamento foi considerado tendendo a ser Casual (somando $n=4$ ), com uma certa aleatoriedade em relação aos elementos. Esta característica pode acarretar problemas principalmente em relação aos princípios de clareza, unidade e simplicidade, bem como na harmonia e proporção. Isto, tendo em vista que a disposição dos elementos não possui uma ordem ou um número limitado de elementos, o que pode atrapalhar o fluxo das informações por ter elementos que podem não ser relevantes ao conteúdo. Podem também prejudicar a atenção dos usuários, direcionando a visão para elementos secundários, o que por consequência pode tornar a retenção do conteúdo mais difícil (memória). No exemplo abaixo, pode-se notar que a disposição dos elementos nos cenários parece ocasionar desequilíbrio das massas gráficas. Além disto, há uso de elementos que podem ser considerados irrelevantes para o conteúdo, e ainda direcionar a atenção dos usuários para estes objetos, distraindo o mesmo.

Figura 5: Capturas de tela do caso: DRC - Atendimento interdisciplinar ao paciente - parte 1. Fonte: UNA-SUS/UFMA, 2016.

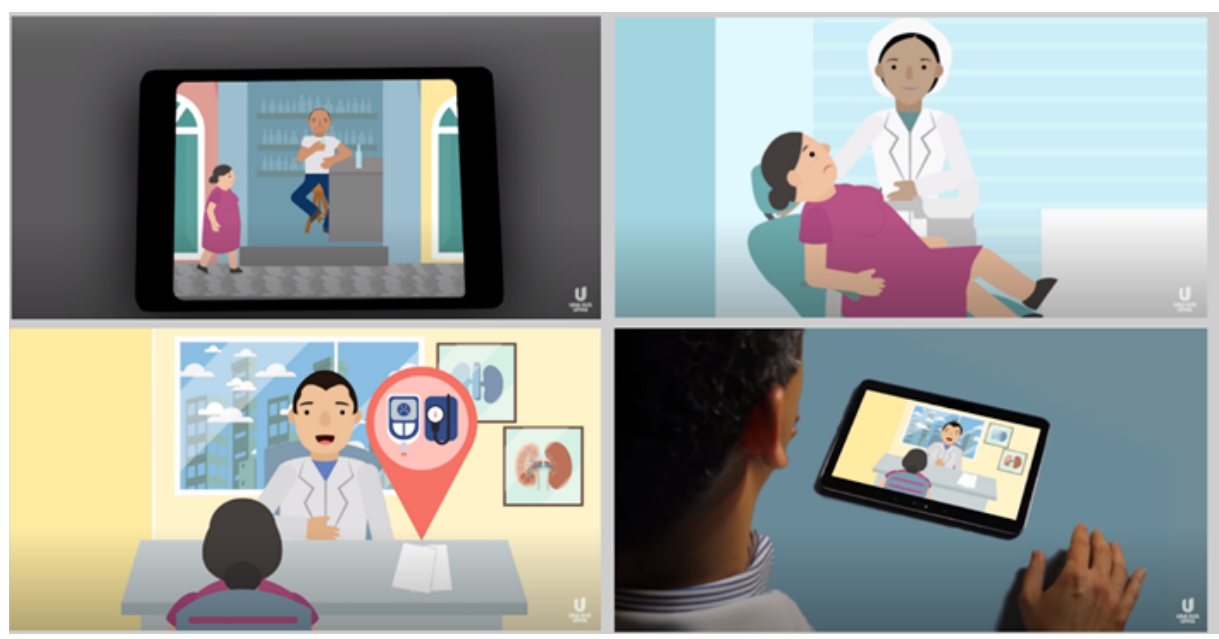


Já a Proximidade foi considerada como sendo neutra $(n=3)$, pois existe uma variação entre a distância da representação e o usuário. Essa característica de estilo é algo que pode influenciar ao princípio da estrutura, em relação a organização e hierarquia do conteúdo. Além disso, a ênfase poderia ser utilizada como recurso de direcionamento da atenção do usuário, através da disposição de elementos mais próximos ou distantes do usuário, não apenas de forma a compor uma cena da animação. A proporção das imagens também pode ser comprometida, no sentido de excessos visuais de elementos em tela. Dessa forma, o valor da proximidade poderia ser utilizado para facilitar a atenção e a percepção dos usuários.

Figura 6: Capturas de tela do caso: DRC - Atendimento interdisciplinar ao paciente - parte 1. Fonte: UNA-SUS/UFMA, 2016.

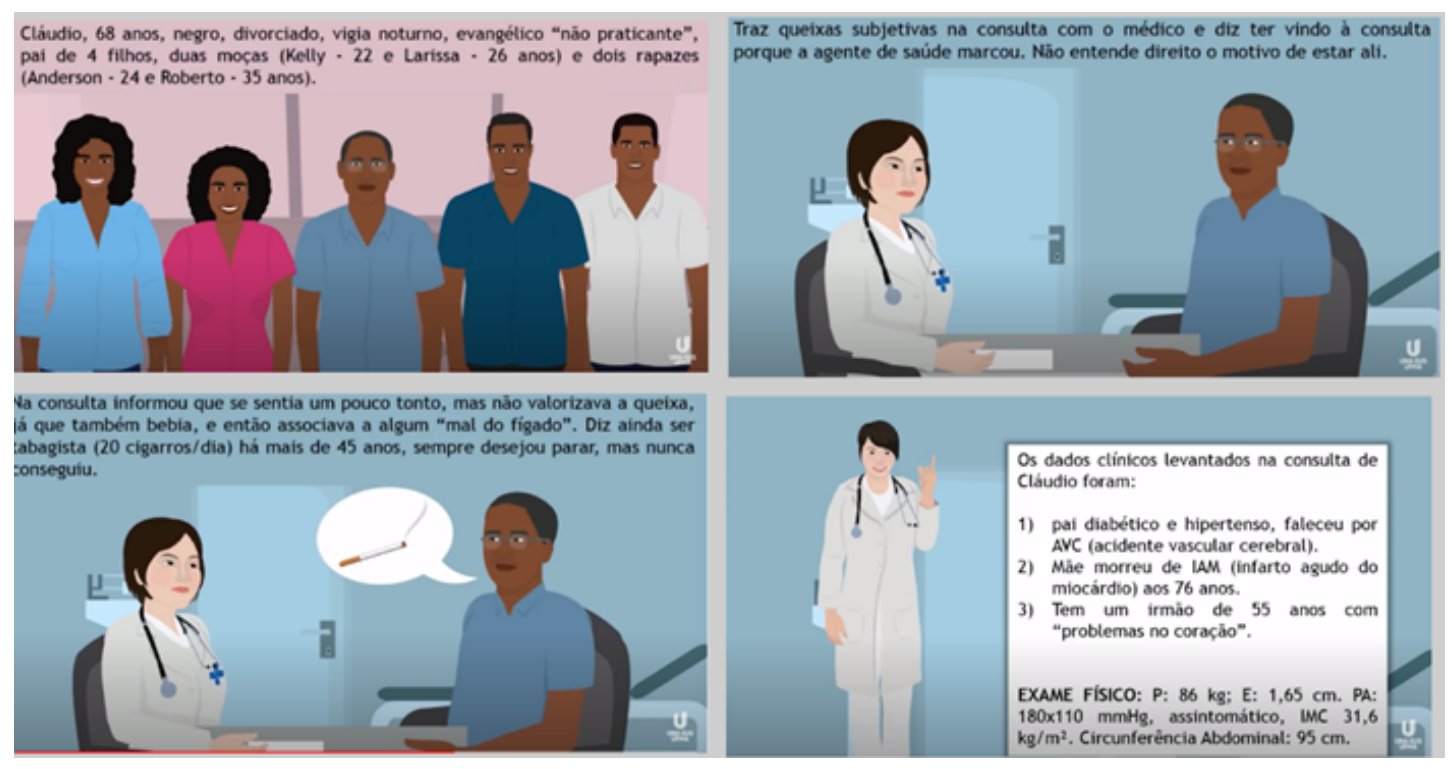

Já a variável Cinética é predominantemente Estática (somando $\mathrm{n}=6$ ), ou seja, com ilustrações sem simulação de movimento, utilizando motion graphics e movimentos de transição como uso do corte seco na transição de cenas e o efeito 'surgir'. A variável Cinética pode influenciar a estrutura, clareza e ênfase da animação, bem como a percepção dos usuários sobre o conteúdo representado. Isto tendo em vista que o movimento pode ser utilizado para chamar a atenção dos usuários. Além disso, não haver movimentação dos personagens nas animações pode gerar estranheza nos usuários, já que o movimento é a base da animação. 
Figura 7: Capturas de tela do caso: Avaliação entre pares: caso Rio de Janeiro.

Fonte: UNA-SUS/UFMA, 2017.

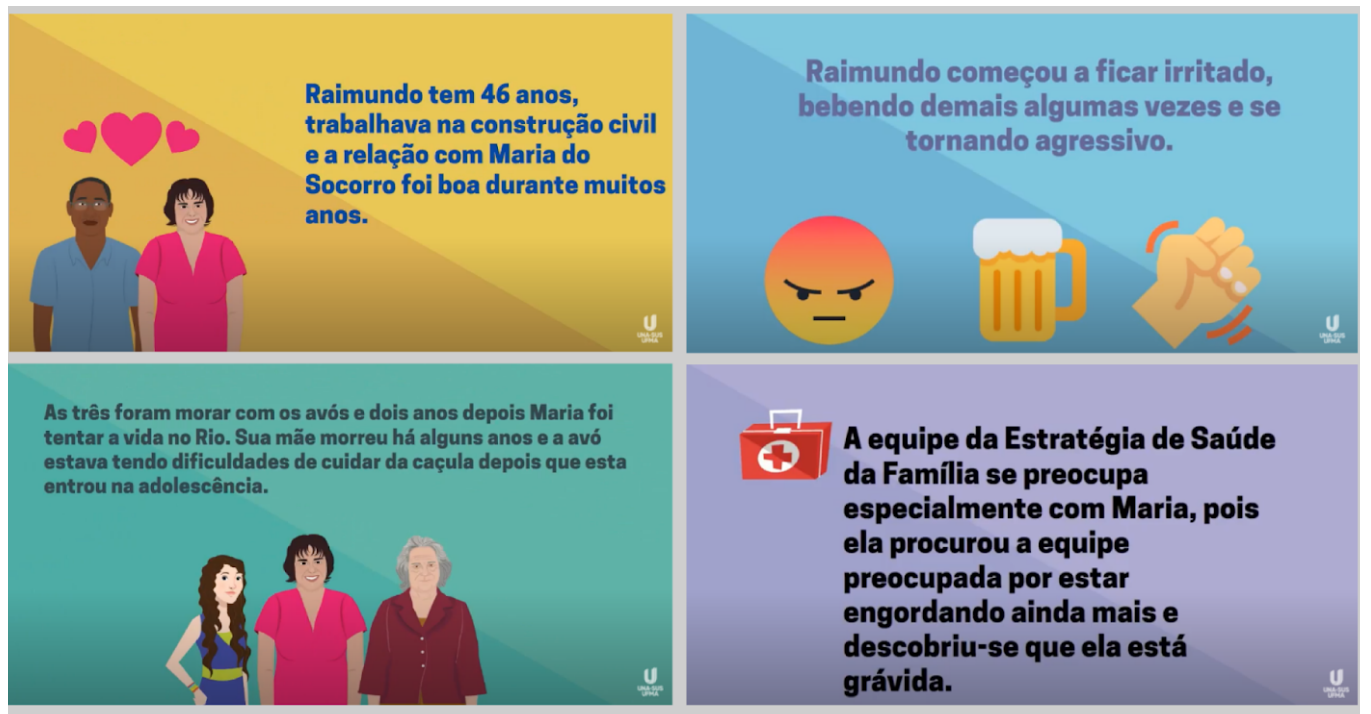

Por fim, o Naturalismo apresentou-se como Não-naturalista (somando $n=6$ ), ou seja, com ilustrações pouco realistas, com características de cartum, ainda que representem situações factíveis. Esta variável tem relação com Consistência e a Gama, pois para a representação não-naturalista tem-se nas animações a técnica de desenho em 2D com pouco detalhamento. Implicações destas variáveis carecem de estudos investigando se os profissionais da saúde sentem-se confortáveis em receber informações através de desenhos em cartum.

Figura 8: Capturas de tela do caso: Caso Clínico Prevenção em DRC. Fonte: UNA-SUS/UFMA, 2016.

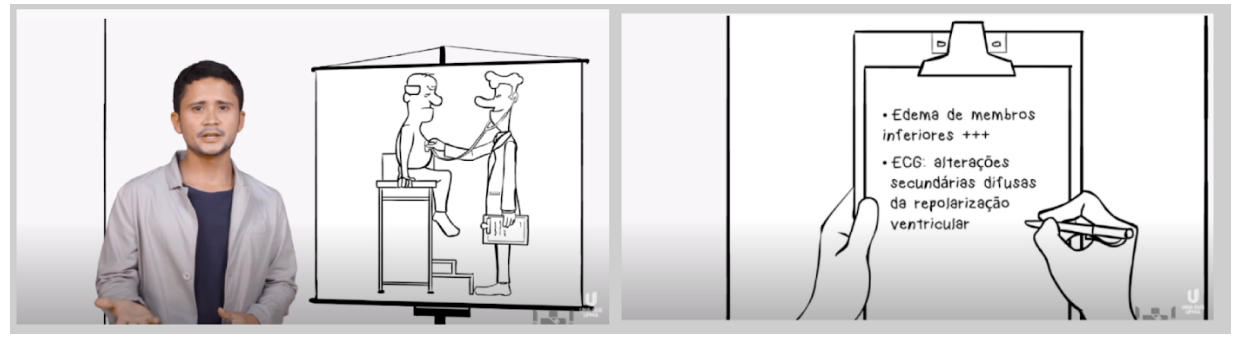

\section{Considerações finais}

Percebe-se que as animações são objetos importantes no contexto do ensino-aprendizagem, sendo utilizadas como recursos educacionais na área da saúde para representar casos clínicos. Estes simulam, situações que auxiliam na capacitação do profissional de saúde na tomada de decisão perante desafios reais do seu dia a dia.

Com a análise das animações aqui apresentadas, foi possível perceber que a UNASUS/UFMA possui um certo padrão estilístico em relação a este recurso educacional. A partir das variáveis de estilo, identificou-se que as animações possuem poucas variações em relação às técnicas de elaboração das ilustrações, com pouco detalhamento, com a apresentação de 
um contexto com certa aleatoriedade em relação à disposição dos elementos. As ilustrações possuem pouca variação de distância em re lação ao usuário, não apresentam simulação de movimento, bem como detalhes que imprimam maior realismo à representação de conteúdos dos casos clínicos.

Relacionando aos princípios propostos por Pettersson (2007) para o design da informação, percebeu-se que nas animações de casos clínicos existe uma simplicidade e unidade pelo uso de ilustrações 2D produzidas por computador, em vetor, sem muitos detalhes, sombra e contraste. Entretanto, destaca-se que as variáveis de estilo podem trazer problemas relacionados à clareza, estrutura, harmonia, proporção. Isto, por muitas das vezes não haver uma diferenciação clara dos elementos representados nas ilustrações: personagens, objetos e cenários podem ser confundidos em sua representação nas cenas. Além disto, não se identificou o uso de recursos de ênfase (e.g., setas, marcações, dentre outros) que poderiam ser interessantes para facilitar a aprendizagem, em aspectos de atenção, percepção, auxiliando o processamento da informação e a memória.

Com base na análise, percebe-se que as características de estilo pictórico são pontos merecedores de atenção em relação ao design das animações de casos clínicos em saúde e devem ser complementas com estudos junto a profissionais de saúde para verificar a eficácia e adequabilidade comunicacional de estilos pictóricos, para que seja possível então a proposição de recomendações para o design de animações sobre casos clínicos.

Por fim, espera-se que as considerações aqui feitas possam contribuir para reflexões sobre estilo pictórico em animações sobre casos clínicos e para apontar oportunidades para futuros estudos em design da informação pictórica em saúde.

\section{Referências}

Ainsworth, S. (2008). How do animations influence learning? In D. Robinson \& G. Schraw (Eds.), Current Perspectives on Cognition, Learning, and Instruction: Recent Innovations in Educational Technology that Facilitate Student Learning. pp. 37-67. Information Age Publishing.

Andrade, R. C. (2014). Infográficos animados e interativos em saúde: Um estudo sobre a compreensão de notícias. Dissertação (Mestrado em Design) - Universidade Federal do Paraná, Curitiba.

Ashwin, C. (1979). The ingredients of style in contemporary illustration: a case study. Information design journal, v. 1, n. 1. p. 51-67.

Dondis, D. A. (1997) Sintaxe da linguagem visual. 2.ed. São Paulo: Martins Fontes.

Galdeano, L. E.; Rossi, L. A.; Zago, M. M. F. (2003). Roteiro instrucional para a elaboração de um estudo de caso clínico. Revista Latino-Americana de Enfermagem, v. 11, n. 3, p. 371-375.

Gombrich, E. H. (1986). Arte e ilusão: um estudo da psicologia da representação pictórica. São Paulo: Martins Fontes, 384p. 
Lima, C. S. C.; Spinillo, C. G.; Assis, K. M. M.; Vital, V. A.; Aquino, I. F. O.; Oliveira, A. E. F. (2019). Análise dos elementos gráfico-informacionais das animações educacionais em Saúde na Educação a Distância (EaD). InfoDesign, v. 16, n. 3, p. 400-418.

Lopes, L. G. A.; Spinillo, C. G. (2017). Variáveis descritivas do estilo de ilustração em sequência pictórica de procedimentos para bulas de medicamentos, p. 1214-1229. In: $16^{\circ}$ Ergodesign. São Paulo: Blucher.

Lowe, R. e Scnhotz, W. (2008). Learning with Animation. Research Implications for Design. Cambridge University Press.

Moore, M.; Kearsley, G. (2007). Educação a Distância: uma Visão Integrada. São Paulo: Thomson Learning.

Oliveira, I. S. C. S.; Coutinho, S. G. (2017). Um olhar sobre o modelo analítico de Clive Ashwin aplicado nas ilustrações de Vera Cruz artista gráfico em Pernambuco - fins do século XIX e início do século XX, p. 1306-1319. In: $8^{\circ}$ CIDI. São Paulo: Blucher.

Pettersson, R. (2007). It Depends: ID - Principles and Guidelines. Tullinge: Institute for Infology.

Spinillo, C. G.; Oliveira A. E.; Mazza, L. U.; Lima, C. S. C.; Assis, K. M. M. (2020) Animações procedimentais em saúde: Proposta de Metodologia de Design para a Universidade Aberta do Sistema Único de Saúde/Universidade Federal do Maranhão. Infodesign. v. 17, n. 3, p. $80-10385$.

Twyman, M. L. (1985). Using pictorial language: a discussion of the dimensions problem. Designing usable texts, p. 245-312.

UNA-SUS. UFMA. Quem somos. 2021. Disponível em: http://www.unasus.ufma.br/site/una-susufma/sobre. Acesso em: 14 de maio de 2021.

\section{Sobre as autoras}

Camila Santos de Castro e Lima, Doutoranda, UFPR, Brasil <camilasclima@gmail.com> Carla Galvão Spinillo, Dra., UFPR, Brasil <cgspin@gmail.com> 